\title{
Hal Oscar Anger, D.Sc. (hon.) (1920-2005): a pioneer in nuclear medicine instrumentation
}

\author{
Hideo Murayama • Tomoyuki Hasegawa
}

Published online: 20 December 2013

(C) Japanese Society of Radiological Technology and Japan Society of Medical Physics 2013

Hal Oscar Anger (Fig. 1) was the inventor of the scintillation camera and one of the pioneers in the field of instrumentation used in nuclear medicine [1-5]. He was born in Denver, Colorado, on May 24, 1920, and grew up in Long Beach, California. He took an interest in the field of electrical engineering, and he received a Bachelor of Science degree in 1943 from the University of California at Berkeley (UCB). Subsequently, he continued his research in radar development at the Radio Research Laboratory of Harvard University until the end of World War II. In 1946, he took up employment at the Donner Laboratory, UCB's Lawrence Radiation Laboratory, and continued to work there until his retirement in 1982. The Donner Laboratory was established by J. Lawrence and C. Tobias for research on the use of radiation in medicine and was named after $\mathrm{W}$. H. Donner, who gave financial support to the laboratory following the loss of his son due to cancer [6]. Initially, Dr. Anger was involved in a research project on a 184-in. cyclotron for use in radiation therapy, but in the 1950s, he began research on instrumentation for the production of images related to the biodistribution of radiopharmaceuticals. He continued his innovative research work in the field of nuclear medicine instrumentation. Dr. Anger received

H. Murayama $(\square)$

Molecular Imaging Center, National Institute of Radiological Sciences, Anagawa 4-9-1, Inage, Chiba 263-8555, Japan e-mail: murayamahideo@gmail.com

T. Hasegawa

School of Allied Health Sciences, Kitasato University, Kitasato 1-15-1, Minami-ku, Sagamihara, Kanagawa 252-0373, Japan e-mail: hasegawa@kitasato-u.ac.jp numerous prestigious awards. He passed away on October 31, 2005, in Berkeley, California.

Research on the use of radioactive tracers was first proposed in 1913 by G. Hevesy, who was awarded the Nobel Prize in Chemistry for his accomplishment. After the end of World War II, research on the medical uses of radiation was encouraged. As short-lived radioactive isotopes such as ${ }^{131}$ I gained broader use, the need for nuclear medicine imaging equipment rose sharply. In 1950, B. Cassen devised the first scintillation scanner [7], which was capable of recording a two-dimensional image of a radioisotope distribution by scanning, namely, passing back and forth a collimator-equipped scintillation counter. However, there was a great demand for a device that could produce images faster without scanning it. In 1952, Dr. Anger integrated a pinhole collimator and a wide $\mathrm{NaI}(\mathrm{Tl})$ scintillator crystal with a photographic plate into a gamma camera that could directly convert the fluorescence created by gamma rays into visible images [8]. This method had drawbacks of a lengthy exposure time and an inability to discriminate between gamma-ray energies.

In the late 1950s, instead of using a photographic plate, Dr. Anger devised a method for estimating the emission position event-by-event by processing the output electrical signals from an array of multiple photomultiplier tubes (PMTs), over which the fluorescence generated from the scintillator is dispersed across a light guide and spread out. A prototype of this gamma camera, referred to as the scintillation camera and later the Anger camera, was constructed successfully and used for imaging of human organs in vivo [9]. In 1958, his epoch-making work was presented at the annual meeting of the Society of Nuclear Medicine (SNM) [10]. The prototype device was equipped with a $\mathrm{NaI}(\mathrm{Tl})$ scintillator 


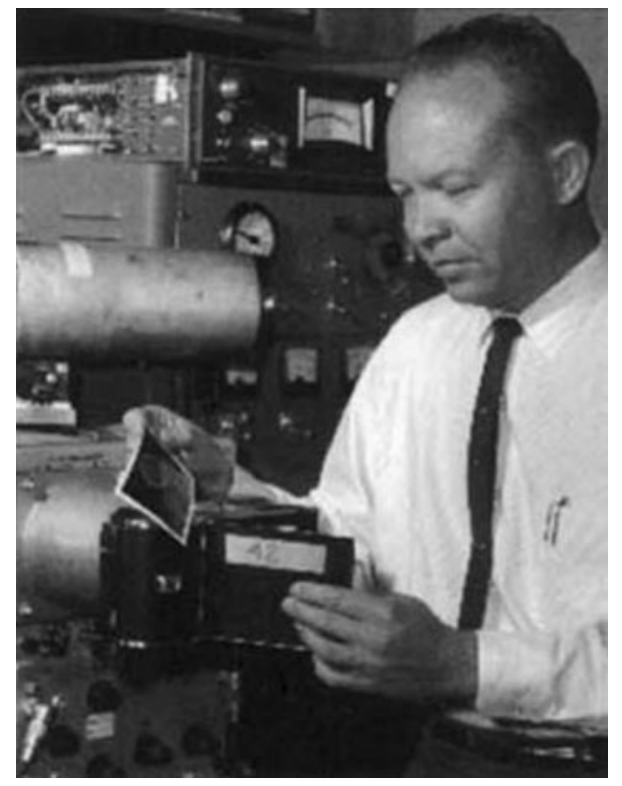

Fig. 1 Portrait of Hal O. Anger, D.Sc. (hon.). Adapted by permission of SNMMI from [2]

that had a diameter of $4 \mathrm{in}$. and a thickness of $1 / 4 \mathrm{in}$., and seven PMTs (Figs. 2, 3, 4, 5). The output of each PMT is weighed by a fixed resistor according to its position in the array. The weighed PMT outputs are summed algebraically to produce a set of $x$ and $y$ coordinate signals that correspond to the location of gammaray interactions in the scintillator. The total output signal from the PMTs was proportional to the gamma ray energy, and thus, the device was capable of discriminating among gamma-ray energies. This made it possible to reduce the effects of scattered gamma rays and, thus, substantially improve the image quality. At about the same time, the ${ }^{99 \mathrm{~m}} \mathrm{Tc}$ generator was first developed, which paved the way for consistently supplying hospitals with short-life radiopharmaceuticals. Consequently, as Anger continued his innovative research and development on the scintillation camera, the nuclear medicine imaging began to exhibit its clinical potential, motivating many researchers and engineers to develop practical and/ or commercial imaging equipment. The scintillation camera has been playing a vital role in the field of nuclear medicine even today. For example, the popular equipment used for single photon emission tomography (SPECT) includes a set of scintillation cameras that are rotated around a subject. In addition, the invention of the scintillation camera also offered great stimuli for other fields such as gamma-ray astronomy.

Dr. Anger's success does not end with the accomplishments outlined above $[1,5]$. In particular, his contributions are of special significance with respect to the

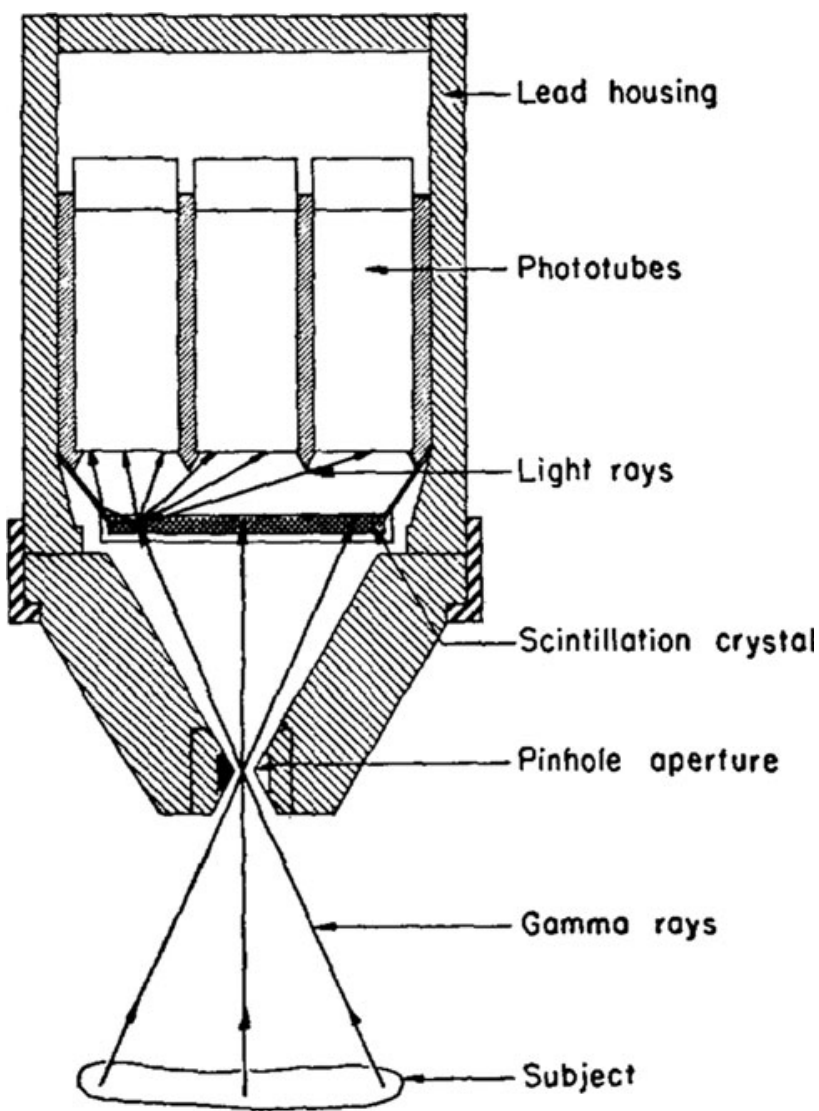

Fig. 2 Sectional drawing of Anger's gamma camera. Reprinted with permission from [9]. Copyright 1958, AIP Publishing LLC

development of the well counter in 1950; the well counter is currently considered to be an indispensable equipment in clinical and research applications. In 1953, he introduced a whole-body scanner, which used ten scintillation counters to scan the whole body. Furthermore, in 1959, he developed the world's first positron camera, in which a pair of scintillation cameras was positioned on opposite sides of a patient and used for coincidence detection of annihilation gamma rays [11]. These accomplishments evolved into more recent successful techniques such as positron emission tomography (PET) and whole-body imaging.

Dr. Anger obtained 15 patents, published numerous papers and books, and was the recipient of various awards, including the John Scott Award in 1964; honorary doctorate in science (D.Sc.) degree from Ohio State University in 1972; a Nuclear Medicine Pioneer Citation from the SNM in 1974; the SNM First Western Regional award in 1976; the Centennial Year Medal from the Institute for Electrical and Electronics Engineers (IEEE) in 1984; the George de Hevesy Memorial Medal, Vienna, in 1991; and the first Cassen Prize from the Education and Research Foundation for the SNM in 1994 [5]. 
Fig. 3 Block diagram of the electrical circuits in Anger's gamma camera. Reprinted with permission from [9]. Copyright 1958, AIP Publishing LLC

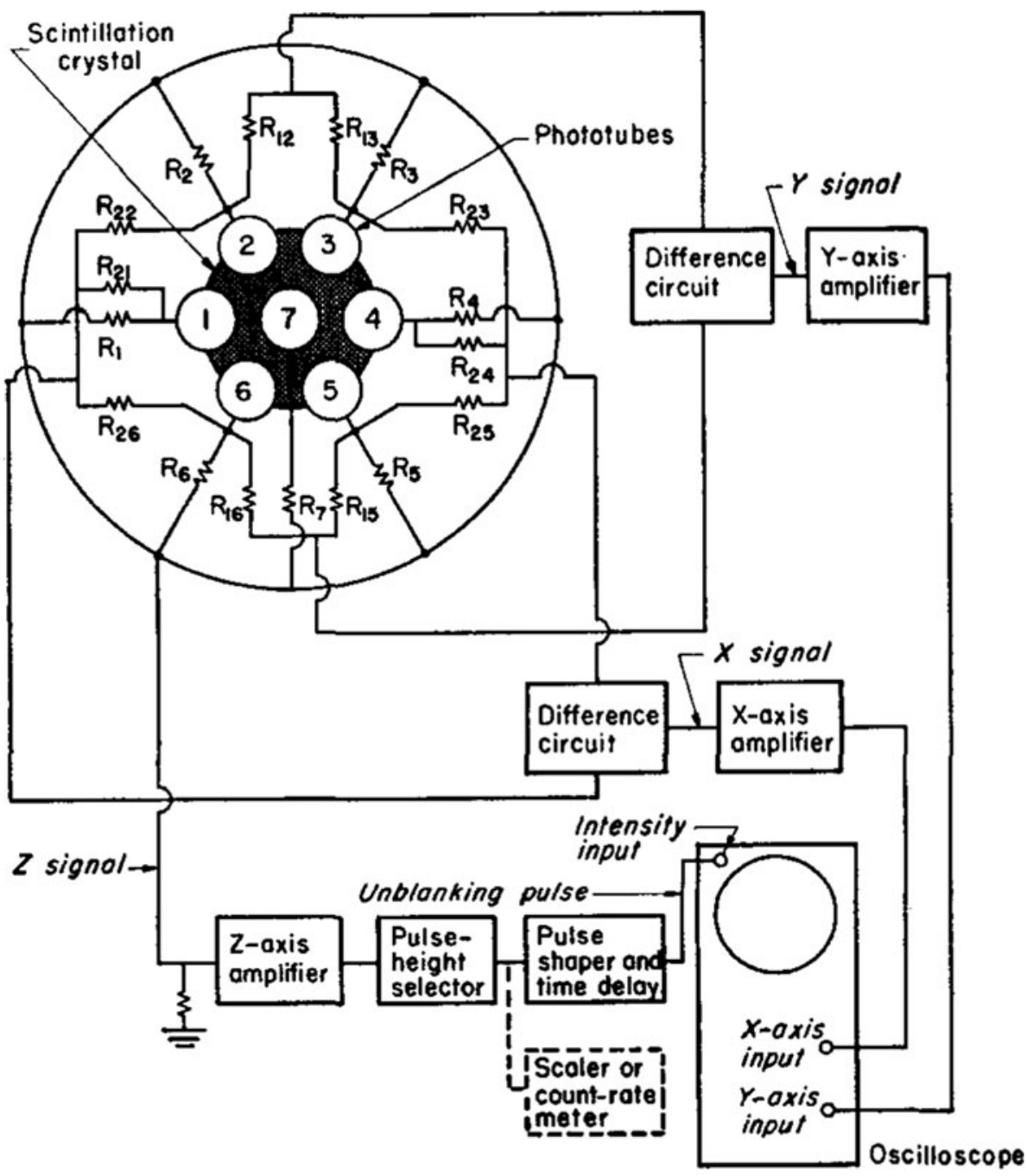

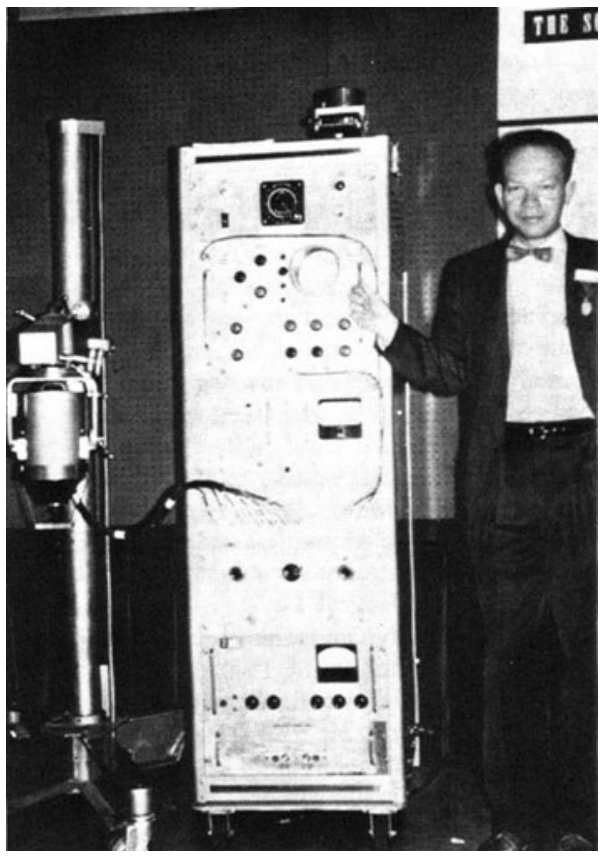

Fig. 4 H. O. Anger and his first gamma camera. Adapted by permission of SNMMI from [10]

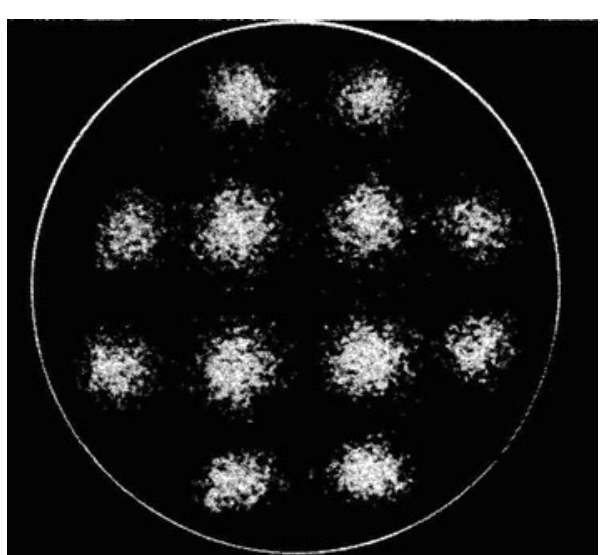

Fig. $5{ }^{131}$ I phantom image from Anger's gamma camera. Reprinted with permission from [9]. Copyright 1958, AIP Publishing LLC 


\section{References}

1. Powell MR. H. O. Anger and his work at the Donner laboratory. Semin Nucl Med. 1978;9:164-8.

2. Tapscott E. Nuclear medicine pioneer: Hal O. Anger. First scintillation camera is foundation for modern imaging systems. J Nucl Med. 1998;39(3):15N, 19N, 26N-27N.

3. Wagner HN Jr. Hal Anger: Nuclear medicine's quiet genius. J Nucl Med. 2003;44(11):26N, 28N, 34N.

4. Gottschalk A. To the newsline editor. J Nucl Med. 2004;45(3):13N-16N, 26N.

5. Tapscott E. Nuclear medicine pioneer: Hal O. Anger, 1920-2005. J Nucl Med. 2005;46(12):11N-12N, 14N, 27N.
6. Williams JE. Donner Laboratory: the birthplace of nuclear medicine. J Nucl Med. 1999;40(1):16N, 18N, 20N.

7. Cassen B, Curtis L, Reed C, Libby R. Instrumentation for ${ }^{131} \mathrm{I}$ use in medical studies. Nucleonics. 1951;9(2):46-50.

8. Anger HO. Use of gamma-ray pinhole camera for in vivo studies. Nature. 1952;170:200-1.

9. Anger HO. Scintillation camera. Rev Sci Instr. 1958;29:27-33.

10. Myers WG. The Anger scintillation camera becomes of age. J Nucl Med. 1979;20(6):565-7.

11. Anger HO. Gamma-ray and positron scintillation camera. Nucleonics. 1963;21(10):56-9. 